\title{
Surgery for Intracranial Tumours in the First Five Years of Life- A Single Institution Consecutive Series of 68 Children
}

\author{
Lene Pernes ${ }^{1}$, Linda Sommerfelt ${ }^{1}$, Tryggve Lundar*, ${ }^{1,2}$ and Finn Wesenberg ${ }^{1,3}$ \\ ${ }^{1}$ Faculty of medicine, University of Oslo, Norway \\ ${ }^{2}$ Department of Neurosurgery, Oslo University Hospital, Rikshospitalet, Norway \\ ${ }^{3}$ Department of Paediatric Medicine, Oslo University Hospital, Rikshospitalet, Norway
}

\begin{abstract}
Thirty-four females and thirty-four males aged 0-4 years were primarily operated for a brain tumour during the years 2005 through 2011. Twenty-two of the tumours were infratentorial and forty-six were supratentorial. Tumours presented more frequently in the first year of life $(n=21)$, and 19 of these infants had a supratentorial tumour. In the 1-4 years age group $(n=47), 27$ children had supratentorial tumours. Twenty-three of the tumours were high-grade tumours, PNET (primitive neuroectodermal tumour) and ependymomas being most frequent. Forty-five were lowgrade, mostly being astrocytomas. The clinical presentation was heterogeneous with a wide range of symptoms.

Conclusions: Tumours presented more frequently in the first year of life (31\%), and $90.5 \%$ of these infants had a supratentorial tumour, in comparison to $57 \%$ in the age group $1-4$ years. Five year overall survival rate was $86,6 \%$ in children with low-grade tumours opposed to $61 \%$ among children with high-grade tumours. The aim of primary surgery with GTR (gross total resection) was achieved in 36 children leading to $94 \%$ overall survival (OS) after 2-8 years follow up. In the 32 children who had only a partial resection or a biopsy, OS was $66 \%$.
\end{abstract}

Keywords: Neurosurgery; Outcome; Paediatric brain tumour; Tumour localisation

\section{Introduction}

Brain tumour in children is fortunately rare, but it is one of the most common cancers among children. Cancer in the central nervous system (CNS) covers approximately one-third of all cancers in children under the age of 15 [1] in Norway. The incidence of brain tumours is 4.2 per 100000 children under the age of 15 [2], or about 40 new cases each year [2,3]. Of these, about 13 children are under the age of $5[1,2]$. In previous publications, children under the age of 15 are mostly reported together. Our aim was to look at age distribution, localisation, and histology in those below the age of five.

\section{Material and Methods}

We retrospectively looked at a consecutive series of 68 patients below 5 years of age primarily operated for intracranial brain tumour at Oslo University Hospital, Rikshospitalet, from 2005 through 2011. The diagnoses were verified by histological examination and the tumours were graded and classified according to the 2007 World Health Organization (WHO) Classification of Tumours of the Central Nervous System [4]. Supratentorial and infratentorial tumours were included, while tumours in the skull and spinal cord were excluded. The study was based on the operative protocol of the neurosurgical department. The last day of follow up was 31/12/13. The following surgical definitions were used: Gross total resection (GTR) indicates absence of any abnormal enhancement on the post-operative Magnetic resonance imaging (MRI) scan, while partial resection indicates more than $10 \%$ of the enhancing lesion left. The information was based on postoperative MRI scans and the surgeons' description.

\section{Statistical methods} [5].

SPSS software, version 18.0 was used to create a Kaplan Meier curve

\section{Clinical Results}

Of the sixty-eight patients there were 34 females and 34 males. Twenty-one patients were below one year of age while forty-seven were between one and four years of age. Of the 1-4 year old children 13 were in the second year of life, 13 were in the third year of life, 10 were in the fourth year of life, and 11 were in the fifth year of life. Comparison with several previous studies is listed in Table 3.

\section{Histology, Age and Localisation}

Forty-five patients had a low-grade tumour classified as WHO classification 1 or 2 . Twenty-three patients had a high-grade tumour corresponding to the WHO classification 3 or 4 . The most common low-grade tumours were astrocytic, while the most common high-grade tumours were embryonic, more specific primitive neuroectodermal tumour (PNET). Distribution of high-grade and low-grade tumours in the supratentorial and infratentorial compartment is shown in Table 1.

Among those below one year 16 had a low-grade and 5 had a highgrade tumour. In the age group 1-4 years 29 had a low-grade tumour and 18 patients had a high-grade tumour. Forty-six tumours were in the supratentorial compartment. Of these, 32 were low-grade while 14 were high-grade. Among the twenty-two infratentorial tumours, 13 were low-grade and 9 were high-grade. Figure 1 shows the distribution between compartments and the tumour grade in the two age groups.

\section{Treatment}

The different treatments for brain tumours available are surgical resections, ventriculoperitoneal shunts, cytostatic drugs, radiotherapy and symptomatic treatment. All the patients received some kind of surgery, either GTR, partial resection or biopsy only . GTR was done in 36 patients while 25 had partial resections. In seven patients only

*Corresponding author: Tryggve Lundar, MD, PhD, Oslo University Hospital HF, Rikshospitalet, Department of Neurosurgery, OSLO, Norway; Tel. +47 2307 43 02; Fax: +47 230743 10; E-mail: tlundar@ous-hf.no

Received August 25, 2014; Accepted October 30, 2014; Published November 07, 2014

Citation: Pernes L, Sommerfelt L, Lundar T, Wesenberg F (2014) Surgery for Intracranial Tumours in the First Five Years of Life- A Single Institution Consecutive Series of 68 Children. Surgery Curr Res 4: 209. doi:10.4172/2161-1076.1000209

Copyright: $\odot 2014$ Pernes L, et al. This is an open-access article distributed under the terms of the Creative Commons Attribution License, which permits unrestricted use, distribution, and reproduction in any medium, provided the original author and source are credited. 
Citation: Pernes L, Sommerfelt L, Lundar T, Wesenberg F (2014) Surgery for Intracranial Tumours in the First Five Years of Life- A Single Institution Consecutive Series of 68 Children. Surgery Curr Res 4: 209. doi:10.4172/2161-1076.1000209

Page 2 of 4

\begin{tabular}{|l|c|c|c|}
\hline SUPRATENTORIAL $(\mathbf{n = 4 6 )}$ & & INFRATENTORIAL (n=22) \\
\hline Low-grade & High-grade & Low-grade \\
\hline Astrocytic tumours $\mathbf{2 1}$ & Oligoastrocytic tumours $\mathbf{1}$ & Astrocytic tumours & $\mathbf{1 0}$ \\
\hline Choroid plexus tumours $\mathbf{4}$ & Embryonal tumours $\mathbf{8}$ & Choroid plexus tumours $\mathbf{1}$ & Astrocytic tumours $\mathbf{1}$ \\
\hline Chraniopharyngioma $\mathbf{2}$ & Ependymal tumours $\mathbf{3}$ & Epidermoid cysts & $\mathbf{2}$ \\
\hline Teratoma $\quad \mathbf{2}$ & Mixed germ cell tumour $\mathbf{1}$ & \\
\hline Neuronal and mixed neuronal-glial tumours $\mathbf{2}$ & Choroid plexus tumour $\mathbf{1}$ & \\
\hline Mesenchymal tumours $\mathbf{1}$ & & \\
\hline
\end{tabular}

Title: Histologic distribution of tumours in 68 children.

Table 1: 2007 WHO classified histology, localisation and tumour grade in 68 children age 0-4 years, treated at the Oslo University Hospital, Rikshospitalet, 2005-2011 [22]

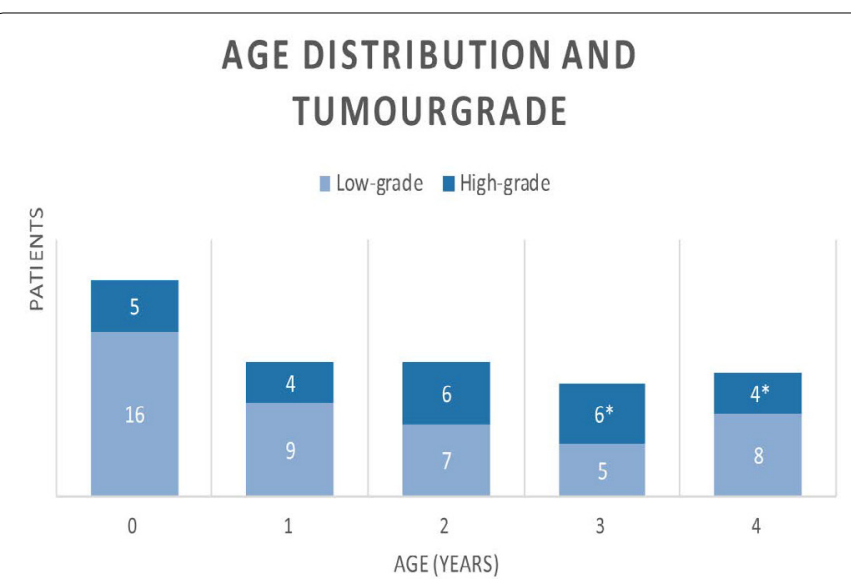

Title: Distribution of tumour grade and localization.

Figure 1: Distribution of tumour grade and localisation in the age groups in 68 patients aged 0-4 years primarily treated for brain tumour at the Oslo University Hospital, Rikshospitalet, 2005-2011.

\begin{tabular}{|l|c|c|c|}
\hline Neurological signs and symptoms & $<\mathbf{1}$ year & $\mathbf{1 - 4}$ years & Total \\
\hline Nausea and vomiting & 6 & 26 & $32(47 \%)$ \\
\hline Altered level of consciousness & 10 & 16 & $26(38 \%)$ \\
\hline Unsteadiness & 1 & 24 & $25(37 \%)$ \\
\hline Headache & 0 & 21 & $21(31 \%)$ \\
\hline Abnormal eye movements & 11 & 8 & $19(28 \%)$ \\
\hline Seizures & 4 & 13 & $17(25 \%)$ \\
\hline Other & 5 & 8 & $13(19 \%)$ \\
\hline Bulging of the fontanelle and splayed sutures & 12 & 0 & $12(18 \%)$ \\
\hline Increased head circumference & 9 & 3 & $12(18 \%)$ \\
\hline Squinting & 2 & 9 & $11(16 \%)$ \\
\hline Cranial nerve palsy & 0 & 10 & $10(15 \%)$ \\
\hline Focal motor weakness & 1 & 7 & $8(12 \%)$ \\
\hline Reduced visual aquity & 3 & 4 & $7(10 \%)$ \\
\hline
\end{tabular}

Title: Neurological signs and symptoms.

Table 2: Neurological symptoms and signs presenting brain tumour in children 0-4 years treated at the Oslo University Hospital, Rikshospitalet, 2005-2011.

biopsies were taken and no further resection was done. Twenty-four of the patients underwent a second resection when indicated. A further three of these had a third resection. In total, the number of surgical resections was 95 during the observation period.

A shunt procedure for cerebral spinal fluid (CSF) diversion was done in 26 patients. Eight of these children had a posterior fossa tumour. Thirty-three had chemotherapy, and nine underwent radiotherapy, all of them older than 20 months of age. Radiotherapy is ideally not used in young children [6], which explains why the number of patients who received this treatment is low.

\section{Signs and symptoms}

The distribution of neurological signs and symptoms of brain tumour in children is displayed in Table 2. Huge variability is shown, and there are some marked differences in the presentation among the youngest children less than one year and the older ones.

\section{Outcome}

Out of 68 children who underwent primary surgery, 55 patients were alive at the end of follow up. All the patients had a followup of at least two years, ranging up to 8 years (from 01/01/2005 to $31 / 12 / 2013$ ). Among the survivors, 40 patients had a low-grade tumour and 15 patients had a high-grade tumour. Thirty-eight tumours had supratentorial and seventeen had infratentorial localisation.

Thirteen patients died, of which eight had supratentorial and five had infratentorial tumours. Eight of these had tumour with high grade of malignancy, while five were diagnosed with a low-grade tumour. Out of the patients with high-grade tumours that died, one was diagnosed in the first year of life, one in the second, three in their third, one in the fourth, and two were in their fifth year of life. They all died within our period of observation from progressive malignant disease. Their histological diagnosis where as follows: six PNET (including two pineoblastoma), one glioblastoma and one anaplastic oligoastrocytoma.

Of the five patients with low-grade tumour that died, two were in their first year of life and three were in their fourth. All of them had astrocytomas, four being optic pathway glioma (supratentorial) and one being located to the brain stem (infratentorial). There are several reasons why these children died; one had a widespread congenital tumour (oncocephalus) taking up most space in the brain, another one had an intrinsic brainstem tumour. The last MRI findings among the dead patients showed 12 with disease in progression, and 1 with a steady residue.

The mortality in children in their first year of life was $14.3 \%$ compared to $20.3 \%$ in the age group $1-4$ years.

The five-year overall survival (OS) rate in our cohort was $78 \%$. It was plotted out as $86,6 \%$ five-year OS among 45 children with lowgrade tumours and $61 \%$ five-year OS among the 23 children with highgrade tumours. This is illustrated by the Kaplan Meier curve (Figure 2), demonstrating a marked difference between the two groups.

Survival was clearly related to the degree of surgical resection. Among the 36 children who underwent a primary GTR, 34 survived (94\%). In the non GTR group (partial resection or biopsy), 21 out of 32 children survived (66\%).

\section{Discussion}

In this series of 68 children almost two-thirds of the patients 
had low-grade tumours. In a consecutive series of 100 children of all ages (0-18 years) in our institution done in $1984-88$ [7], only $58 \%$ of the children had low-grade tumours. The increased proportion of low-grade today compared to 30 years ago may be related to a more extensive use of MRI.

In this study, the male/female ratio (M/F ratio) was 1.0. In the series done 30 years ago [7], the M/F ratio was 1.5 , while in the Lannering study from Sweden [8], the M/F ratio was 1.1. Most clinical series of paediatric brain tumours show an excess of males (Table 3 ).

Posteror fossa tumors has been considered typical for pediatric brain tumors [9-11].

An increase in tumours located supratentorially has been observed in our institution during the last decades. In the series done in 1984-88 [7] roughly half of the tumours were supratentorial, compared to $68 \%$ in our series. The age distribution nevertheless has remained the same, the trend indicating that children below 12 months most often present with supratentorial tumours. The present series enhances this shift,

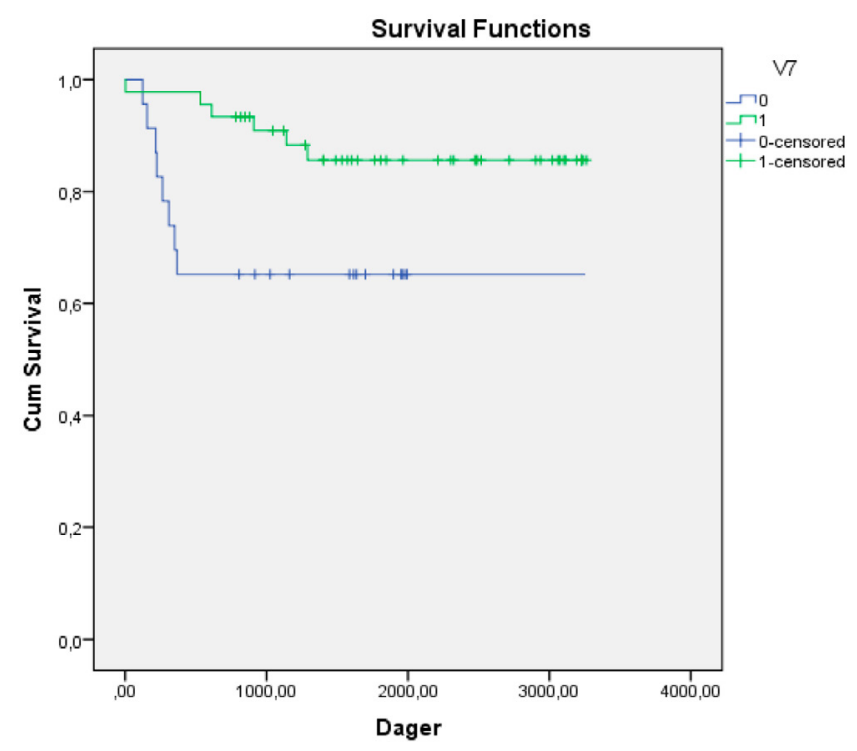

Title: Survival rate

Figure 2: A Kaplan Meier curve showing survival rate in days over time in 68 children age 0-4 years treated at the neurosurgical department, Oslo University Hospital, Rikshospitalet, 2005-2011. The green (black) line is showing patients with low-grade tumours, while the blue (grey) line shows patients with high-grade tumours. especially for small children in their first year of life. We also found a clear predominance of brain tumours presenting in the first year of life (31\%), relative to $26 \%$ in the study from 1990 [7]. In a study from Sweden [8] only $16 \%$ of children under the age of 5 presented in the first year of life. The relative predominance of infants (0-12 months) with supratentorial and mostly low-grade tumours is in contrast to previous studies $[12,13]$.

In our study, nine to ten children under 5 years underwent primary surgery each year in the period 2005-2011. This is an increase compared to the series from 1990 [7], were only five to six children were operated each year. The institution obtained an MRI machine in 1987 and this may explain why the number of primary surgeries has increased. In addition to surgery, $49 \%$ of the patients received chemotherapy, and $13 \%$ underwent radiotherapy. Radiotherapy is ideally avoided in children, especially in those under the age of five, due to the risk of growth failure and other long-term side effects, which are more pronounced in children than in adults [5,14]. In high-grade tumours though, chemotherapy and radiotherapy are important adjunctive therapies and must be considered in each case.

The survival in children with brain tumour is highly dependent on the histology of the tumour, having a high-grade tumour being consistent with a poorer prognosis $[8,10,11,15]$. This is verified in our material with a 5 -year OS rate being $86,6 \%$ among the patients with low-grade tumour and $61 \%$ among the high-grade tumour patients. In spite of the short observation period in this cohort it is a striking difference. Furthermore, the importance of GTR was clear, as OS was $94 \%$ compared to $66 \%$ in children with a more restricted resection. The importance of GTR has been underscored in many previous studies $[11,15]$ and our results are in accordance with institutional as well as national and international series of patients [15].

Our study also revealed differences in mortality among the different age groups, being $14.3 \%$ in the first year of life compared to $20.3 \%$ in the 1-4 year old children. Lannering et al. [8] found that infants below 12 months had a significantly inferior prognosis compared to older children. This may be due to congenital brain tumours. In our series, however, the mortality was higher in the age group 1-4 years, consistent with the higher proportion of malignant tumours.

The predominance of tumour presentation in the first year of life in our cohort may indicate that these children were diagnosed early and treated accordingly, giving them a better outcome. Children below 5 years of age combined seem to have the same prognosis as older children with a 5 -year OS rate estimated to $78 \%$ in the children below 5 years, and being $77.1 \%$ in children below 15 years of age in Norway [16].

\begin{tabular}{|c|c|c|c|c|c|c|}
\hline Reference No. & Author & Age Group (Year) & Male/Female ratio & Low Grade (\%) & High Grade (\%) & Infratentorial (\%) \\
\hline Present study & & $0-4$ & 1 & 66 & 34 & 32 \\
\hline 7 & Lundar et al. & $0-18$ & 1,5 & & & \\
\hline 17 & Nejat et. al. & $0-14$ & & & & $60-70$ \\
\hline 8 & Lannering et al. & $0-14$ & 1,11 & 65 & 35 & \\
\hline 18 & Gjerris et al. & $0-14$ & 1,15 & 56 & 44 & 54 \\
\hline \multirow[t]{2}{*}{19} & $\begin{array}{c}\text { Raaschou-Nielsen } \\
\text { et al. }\end{array}$ & $0-4$ & 0,98 & 63 & approx. 37 & \\
\hline & & $0-14$ & 1,17 & 60 & approx. 40 & 60 \\
\hline \multirow[t]{2}{*}{20} & Mathew et al. & $0-3$ & & 32 & 68 & \\
\hline & & $0-14$ & & 42 & 58 & \\
\hline 22 & Di Rocco et al. & $0-1$ & 1,22 & 47 & 53 & 41 \\
\hline
\end{tabular}

Table 3: Comparison with previous studies. 
In our study we had five children with low-grade tumour that died. Concerning brain tumour, both benign and malignant tumour types are classified as cancer, one of the reasons being that the tumour localisation can make the treatment difficult in which again can affect the prognosis [17]. Even if low-grade tumours in general have a much better prognosis than high-grade tumours, and children usually tolerate surgery very well, some will still not survive a low-grade tumour [18]. This was the case in a boy with a low-grade glioma in medulla oblongata. After biopsy he underwent chemotherapy and radiotherapy. He nevertheless succumbed from progressive disease. As opposed to adults, tumours in children often consist of immature, undifferentiated tumour cells [18]. Optic pathway gliomas can show huge variability in growth and aggressiveness. They are often congenital, widespread and can grow tremendously, ultimately threatening vital functions of the brain [18].

Brain tumours in children below the age of 5 show a varied clinical presentation. The initial symptoms may be subtle or can mimic other more common childhood conditions, such as gastroenteritis [19] that may lead to delayed diagnosis. It is important to keep in mind the development in younger children, especially considering the closure of the fontanelles by 18 months of age, as well as the continuous development of the brain system and function. For instance the increase in head circumference compensates for the increased volume of the brain, postponing the clinical symptoms of high intracranial pressure [18]. The clinical presentation in our cohort is consistent with what is found in earlier literature $[12,19,20]$. We could not confirm the diagnostic delay in children with brain tumours presented by Wilne et al. [21].

The strength of our study is the consecutive series of patients over seven years. All patients have been identified from the protocol of surgery and no one has been excluded, or lost to follow up.

The short observation period in this study does not give a good description of long term results, neither does it describe the quality of life among the survivors, this was however not the focus of the study.

\section{Conclusion}

Our data revealed some important differences regarding tumour presentation among children below 5 years of age, especially an increased incidence of low-grade tumours with supratentorial localisation in the first year of life. Gross total resection of the tumour clearly improved survival.

Almost two-thirds of the patients had low-grade tumours. A greater proportion of low-grade tumours in this study compared to earlier studies could be explained by the extended use of MRI. The histological distribution on our series is remaining equivalent to other European countries.

Concerning prognosis it has been confirmed that GTR as well as histology of the tumour are factors that makes most impact, highgrade tumours having a poorer outcome looking at survival in a 5-year interval.

Children less than one year of age present with a different clinical picture than the children 1-4 years, this may be partially due to the continuously developing brain system.

\section{Compliance with Ethical Standards}

Lene Pernes and Linda Sommerfelt equally contributed to extraction of clinical data. All authors contributed to planning, drafting, writing and discussion of the manuscript.

The authors declare that they have no conflict of interest.

\section{No specific funding.}

Concerning human rights this is a retrospective study were formal consent is not required.

This article does not contain any studies with animals performed by any of the authors.

\section{References}

1. Cancer Registry of Norway (2013) Cancer in Norway 2011 - Cancer incidence mortality, survival and prevalence in Norway.

2. Lie S (2001) Progress in the field of paediatric oncology. Tidsskr Nor Laegeforen 121: $951-955$

3. http://www.ssb.no/a/barnogunge/2011/tabeller/befolkning/bef0000.html.

4. Louis D, Ohgaki H, Wiestler O, Cavenee W, Burger P, et al. (2007) The 2007 WHO Classification of Tumours of the Central Nervous System. Acta Neuropathol 114: 97-109.

5. SPSS Inc. (2009) PASW Statistics for Windows, Version 18.0. Chicago: SPSS Inc

6. Syndikus I, Tait D, Ashley S, Jannoun L (1994) Long-term follow-up of young children with brain tumours after irradiation. Int J Radiat Oncol Biol Phys 30: 781-787.

7. Lundar T, Langmoen IA, Hovind KH, Skullerud K (1990) Brain tumours in childhood. Tidsskr Nor Laegeforen 110: 1809-1811.

8. Lannering B, Sandström PE, Holm S, Lundgren J, Pfeifer S, et al. (2009) Swedish Childhood CNS Tumor Working Group (VCTB) (2009) Classification, incidence and survival analyses of children with CNS tumours diagnosed in Sweden 1984-2005. Acta Paediatr 98: 1620-1627.

9. Nejat F, El Khashab M, Rutka JT (2008) Initial management of childhood brain tumors: Neurosurgical Considerations. J Child Neurol 23: 1136-1148.

10. Gjerris F, Agerlin N, Borgesen SE, Buhl I, Haase J, et al. (1998) Epidemiology and prognosis in children treated for intracranial tumors in Denmark 19601984. Childs Nerv Syst 14: 302-311.

11. Raaschou-Nielsen $O$, Sørensen $M$, Carstensen $H$, Jensen $T$, Bernhardtsen $T$,et al. (2006) Increasing incidence of childhood tumours of the central nervous system in Denmark, 1980-1996. British J Cancer 95: 416-422.

12. Haddad S, Menezes A, Bell W, Godersky J, Afifi A, et al. (1991) Brain tumors occuring before 1 year of age: a retrospective review of 22 cases in an 11-year period (1977-1987). Neurosurgery 29: 8-13.

13. Di Rocco C, Ceddia A, lanelli A (1993) Intracranial Tumour in the First Year of Life. A Report on 51 cases. Act Neurochir (Wien) 123: 14-24.

14. Fossen A, Skjeldal OH, Storm-Mathisen I (1989) Psychological and neurologic long term effects of brain tumour in children. Tidsskr Nor Laegeforen 109: 3406 3408.

15. Mathew RK, O`Kane R, Parslow R, Stiller C, Kenny T, et al. (2014) Comparison of survival between the UK and US after surgery for most common pediatric CNS tumors. Neuro Oncol 16: 1137-1145.

16. Kolmannskog S, Wesenberg F, Lundar T, Lote K (2009) Solide svulster hos barn. (edn $7^{\text {th }}$ ) Cytostatikaboken ,Farmakologisk institutt, Det medisinske fakultet, Universitet i Oslo.

17. Helseth E, Due-Tønnessen BJ, Lundar T, Scheie D, Skullerud K, et al. (2003) Intracranial tumours in children. Tidsskr Nor Laegeforen 123: 451-455.

18. Ching L, Wan-Tee T (2014) The clinical manifestations and diagnosis of central nervous system tumors in children. UpToDate - a evidence-based clinical decision support resource.

19. Wilne SH, Ferris RC, Nathwani A, Kennedy CR (2006) The presenting features of brain tumors: a review of 200 cases. Arch Dis Child 91: 502-506.

20. Tomohisa F, Tabuchi A, Adachi Y, Mizumatsu S, Tamesa N, et al. (1998) Primary brain tumours in children under age 3 years. Brain Tumor Pathol. 15: 7-12.

21. Wilne S, Collier J, Kennedy C, Jenkins A, Grout J, et al. (2012) Progression from first symptom to diagnosis in childhood brain tumours. Eur J Pediatr 171 87-93

22. Rickert C, Paulus W (2001) Epidemiology of central nervous system tumours in childhood and adolescence based on the new WHO classification. Childs Nerv Syst 17: 503-511. 